\title{
Photoinduced modulation of the excitonic resonance via coupling with coherent phonons in a layered semiconductor
}

\author{
S. Mor $\odot,{ }^{1,2}$ V. Gosetti, ${ }^{1,2,9}$ A. Molina-Sánchez $\odot,{ }^{3}$ D. Sangalli, ${ }^{4,6}$ S. Achilli $\odot,{ }^{5,6}$ V. F. Agekyan $\odot,{ }^{7}$ P. Franceschini, ${ }^{1,2,8}$ \\ C. Giannetti ${ }^{1,2}$ L. Sangaletti, ${ }^{1,2}$ and S. Pagliara ${ }^{1,2, *}$ \\ ${ }^{1}$ Department of Mathematics and Physics, Università Cattolica, I-25121 Brescia, Italy \\ ${ }^{2}$ Interdisciplinary Laboratories for Advanced Materials Physics (I-LAMP), Università Cattolica, I-25121 Brescia, Italy \\ ${ }^{3}$ Institute of Materials Science (ICMUV), University of Valencia, Catedrático Beltrán 2, E-46980 Valencia, Spain \\ ${ }^{4}$ Istituto di Struttura della Materia-CNR (ISM-CNR), Division of Ultrafast Processes in Materials (FLASHit), Area della Ricerca di Roma 1, \\ Monterotondo Scalo, Italy \\ ${ }^{5}$ Dipartimento di Fisica, Università degli Studi di Milano, via Celoria 6, 20133 Milano, Italy \\ ${ }^{6}$ European Theoretical Spectroscopy Facility, ETSF \\ ${ }^{7}$ St. Petersburg State University, St. Petersburg, 199034, Russia \\ ${ }^{8}$ Department of Physics and Astronomy, KU Leuven, Celestijnenlaan 200D, 3001 Leuven, Belgium \\ ${ }^{9}$ Department of Materials Engineering, KU Leuven, Kasteelpark Arenberg 44, 3001 Leuven, Belgium
}

(Received 18 May 2021; accepted 3 November 2021; published 13 December 2021)

\begin{abstract}
The coupling of excitons with atomic vibrations plays a pivotal role on the nonequilibrium optical properties of layered semiconductors. However, how exciton-phonon coupling manifests in the time and energy domains is still an open debate between experiment and theory. By means of time-resolved broadband optical reflectivity combined with $a b$ initio calculations of a bismuth tri-iodide single crystal, we set the spectral fingerprints for the optical detection of exciton-phonon coupling in layered semiconductors. Our joint experimental and theoretical effort allows us to unravel the impact of exciton-phonon coupling by microscopically relating the photoinduced coherent energy modulation of the excitonic resonance to coherent optical phonons. This enables us to track the extent of the photoinduced atomic displacement in real space. Our findings represent a step forward on the road to coherent manipulation of the excitonic properties on ultrafast timescales.
\end{abstract}

DOI: 10.1103/PhysRevResearch.3.043175

\section{INTRODUCTION}

Electron-phonon coupling is among the fundamental interactions in condensed matter which govern the nonequilibrium optoelectronic properties of materials by, for instance, guiding the relaxation dynamics of quasiparticles [1-4] and assisting nonthermally driven electronic phase transitions [5-7]. In semiconductors, the electron-hole Coulomb interaction also plays a crucial role in allowing the formation of bound states (excitons) upon photoabsorption. The binding energy of excitons is usually of a few meV, making them unstable at room temperature and difficult to be detected. However, in layered semiconductors, the reduced dielectric screening strongly enhances the exciton binding energy up to several hundreds of $\mathrm{meV}$ [8]. This fact, together with the technological relevance of layered materials, is triggering enormous research efforts in capturing exciton dynamics on the femto- to picosecond timescale [9-15]. Recently, exciton-phonon coupling has been proposed to impact on not only the incoherent carriercooling dynamics, but also the homogeneous linewidth of the

\footnotetext{
*Corresponding author: stefania.pagliara@unicatt.it

Published by the American Physical Society under the terms of the Creative Commons Attribution 4.0 International license. Further distribution of this work must maintain attribution to the author(s) and the published article's title, journal citation, and DOI.
}

excitonic luminescence, and even the diffusion and the coherence length of excitons $[14,16]$. However, the microscopic mechanism by which a lattice vibration interacts with the excitons has not found a description yet, which is corroborated by both experiment and theory [10-12,14,17].

While access to exciton-phonon coupling is a great challenge both experimentally and theoretically, discerning and controlling its effects on the nonequilibrium optical response are of paramount interest from a fundamental viewpoint, as well as for the technological progress. Experimentally, resonant Raman scattering spectroscopy is among the most common techniques to address exciton-phonon coupling $[17,18]$. However, the exciton dynamics remain out of reach due to the lack of temporal resolution. Time-resolved optical spectroscopy is the most suitable tool to investigate the interaction between excitons and phonons in the ultrafast time domain $[11,15,19,20]$. By measuring transient changes in the optical polarization, the technique provides macroscopic snapshots of the nonequilibrium behavior of the system. On the theoretical side, $a b$ initio calculations of excitonphonon coupling combine density-functional-perturbation theory for the electron-phonon matrix elements [21], with the Bethe-Salpeter equation (BSE), which correctly describes the excitonic physics [22]. Such scheme has been recently developed and applied to two-dimensional materials to compute exciton-phonon lifetimes [14,23]. An alternative approach computes the excitonic properties solving the BSE within the 
$\mathrm{GW}$ approximation both at equilibrium and with the atoms displaced along specific phonon modes [11]. This method further enables an estimate of atomic displacements with exceptionally high spatial resolution on the subpicometer scale [24].

In this Article, by combining time-resolved broadband optical reflectivity measurements with state-of-the-art spinorial $a b$ initio BSE calculations [25], we unveil the spectral fingerprints of exciton-phonon coupling in a representative van-der-Waals layered semiconductor, the bismuth tri-iodide $\left(\mathrm{BiI}_{3}\right)$ single crystal. Recently, $\mathrm{BiI}_{3}$ has come into focus as an efficient nontoxic replacement of lead-based perovskites in optoelectronic applications [26,27]. The absorption spectrum is dominated by a direct excitonic resonance at $620 \mathrm{~nm}$ $(2.00 \mathrm{eV})$ with lifetime of nanoseconds and binding energy of hundreds of meV [28]. Such large binding energy in a bulk semiconductor is a rather unique feature. Resonant Raman scattering combined with luminescence have identified an interaction between excitons and the main $A_{g}$ vibrational mode at $3.4 \mathrm{THz}\left(113 \mathrm{~cm}^{-1}\right)$ [29-32]. More recently, transient absorption of spin-coated thin films of $\mathrm{BiI}_{3}$ observed a combined dynamics of coherent optical phonons and excitons upon ultrafast photoexcitation [33]. All these aspects make $\mathrm{BiI}_{3}$ an ideal platform for exploring experimentally and theoretically the photophysics of excitonphonon coupling in a rather straightforward manner compared to more complex systems $[15,20]$. However, both the spectral fingerprint of the coupling and its impact on the exciton and phonon dynamics have remained elusive until now, likely due to the difficulty in providing univocal proof both experimentally and theoretically.

Here, we provide evidence that exciton phonon coupling sets in at the photoexcitation time and triggers a coherent modulation of the excitonic resonance of $\mathrm{BiI}_{3}$ resulting from a combination of a single-particle band gap renormalization and a counteracting change in the exciton binding energy. The displacive photoexcitation of coherent optical phonons produces oscillations of the transient reflectivity which are enhanced by almost one order of magnitude at wavelengths close to the excitonic resonance. Moreover, the exciton energy varies periodically within a few picoseconds, in phase with the coherently generated atomic vibrations. This is captured by retrieving the initial phase of the coherent phonon amplitude across the exciton wavelength and monitoring the energy shift of the excitonic peak. The ab initio calculations corroborate that all observations are univocal fingerprints, which could be used to reveal and study exciton-phonon coupling also in other materials. Moreover, our joint experimental and theoretical approach provides a unique tool to address exciton-phonon coupling in real space by retrieving the extent of the atomic displacement responsible for the coherent modulation of the excitonic resonance.

\section{TRANSIENT REFLECTIVITY DATA AND AB INITIO SIMULATIONS}

The photoinduced transient reflectivity change, $\left(\Delta R / R_{0}\right)(t)$, is shown as color scale in Fig. 1(a) as a function of probe wavelength (left axis), probe photon energy (right axis), and pump-probe time delay (top axis).

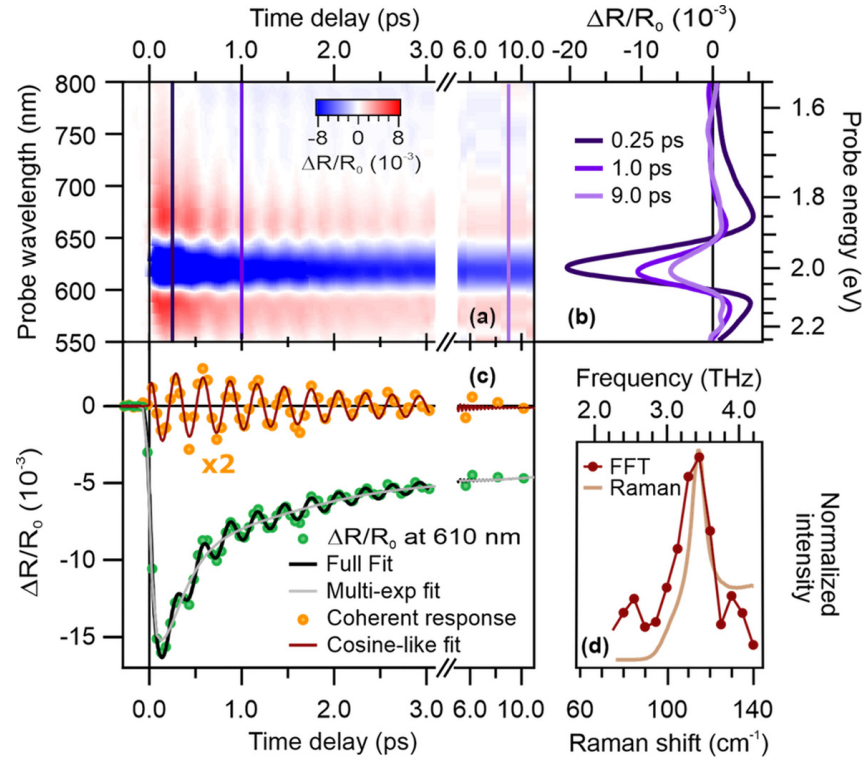

FIG. 1. (a) Measured $\Delta R / R_{0}$ as a function of probe wavelength and pump-probe time delay. (b) Vertical line cuts of (a) at selected time delays of $0.25,1.0$, and $9.0 \mathrm{ps}$. (c) Horizontal line cut of (a) at $610 \mathrm{~nm}$ (green circles) superimposed by its full-fit curve (black line). The multiexponential fit contribution (gray line) is subtracted from the data to obtain the bare coherent optical response (orange circles) shown together with the relevant fit (red line). (d) FFT of the coherent optical response at $610 \mathrm{~nm}$ (red circles) and Raman scattering spectrum (light brown line).

The measurement is carried out at room temperature and the pump photon energy is $2.78 \mathrm{eV}(445 \mathrm{~nm})$, well above the band gap of $\mathrm{BiI}_{3}$. This enables the excitation of $n_{c}=$ $2 \times 10^{19} \mathrm{el} / \mathrm{cm}^{3}$ quasifree carriers into the conduction bands (see [34] for the calculation). At positive time delays, i.e., after photoexcitation, a pronounced negative (blue) signal with minimum at $620 \mathrm{~nm}(2.00 \mathrm{eV})$ indicates a transient decrease of reflectivity $\left[\left(\Delta R / R_{0}\right)(t)<0\right]$ in the spectral range of the excitonic resonance. Above and below this spectral region, the signal is positive (red), as the transient reflectivity increases $\left[\left(\Delta R / R_{0}\right)(t)>0\right]$. The temporal evolution of both the negative and positive spectral features is emphasized by the spectra reported in Fig. 1(b) for selected time delays. At all probe wavelengths, this incoherent optical response is superimposed by a periodic intensity modulation [see, e.g., the coherent response at $610 \mathrm{~nm}$ in Fig. 1(c)] resulting from the generation of coherent optical phonons by the pump pulse.

The transient reflectivity signal at each wavelength is fitted by a sum of four exponentially decaying functions (incoherent optical response) and an exponentially decaying cosine function (coherent optical response) convoluted with a Gaussian function (pump-probe cross-correlation) (see [24] for the fit function). Figure 1(c) shows the fit (black line) for the data at $610 \mathrm{~nm}$ (green circles). The gray line is the multiexponential fit contribution to the incoherent optical response, only. At all wavelengths, we find that the incoherent optical response decays through multiple relaxation channels with time constants on the order of $200 \mathrm{fs}$, few picoseconds, hundreds of picoseconds, and $1.5 \mathrm{~ns}$, respectively. These values agree with previous measurements of the 
transient absorption of spin-coated $\mathrm{BiI}_{3}$ thin films [33] and the luminescence of $\mathrm{BiI}_{3}$ single crystals [35]. Accordingly, we assign the fastest dynamics to carrier-carrier scattering, and the two intermediate dynamics to electron-phonon scattering processes and the beginning of electron-hole recombination. The slowest dynamics exceed the investigated temporal window and its time constant has been held during the fitting procedure to the literature value [35] of the electron-hole radiative recombination process.

After subtraction of the multiexponential fit component [gray line in Fig. 1(c)], the coherent optical response (orange circles) is retrieved. Its fast Fourier transform [FFT, red circles in Fig. 1(d)] reveals an oscillation at $3.41 \pm 0.02 \mathrm{THz}$ matching the frequency of the $A_{g}$ phonon mode (light brown line) [32]. Moreover, the oscillation intensity is damped, on average, within $1.860 \pm 0.075 \mathrm{ps}$, which provides the dephasing time of the coherent phonons.

To shed light on the nature of the transient optical response, we perform numerical analysis of the optical properties of $\mathrm{BiI}_{3}$ via $a b$ initio simulations within the $\mathrm{GW}+\mathrm{BSE}$ scheme (see [34] for details about the theoretical model). As shown in Fig. 2(a), the imaginary part of the dielectric function is dominated, in the optical region investigated in our measurements, by an excitonic peak with binding energy of $500 \mathrm{meV}$ [36]. Such large binding energy is consistent with the strong in-plane localization of the exciton wave function [37] shown in the real-space representation of Fig. 2(b). The computed reflectivity spectrum, $R^{X_{0}}(\omega)$ [thin line in inset of Fig. 2(a)] exhibits a resonance at the energy of the excitonic peak. To match $R^{X_{0}}(\omega)$ with the experimental reflectance spectrum (thick line), the calculated peaks are shifted here by $0.35 \mathrm{eV}$. Thus, our calculations clearly show that the optical reflectivity is mostly dominated by the imaginary part of the dielectric function, in agreement with previous works $[38,39]$. We then interpret the incoherent optical response as follows: The negative peak at $620 \mathrm{~nm}(2.00 \mathrm{eV})$ is assigned to the pump-induced photobleaching of the excitonic resonance; the positive signal at shorter and longer wavelengths to a combination of photoabsorption between transiently excited states, screeninginduced optical gap renormalization [33], and increase of the exciton linewidth.

We now focus on the bare coherent optical response of $\mathrm{BiI}_{3}$. The frequency-resolved coherent optical phonon spectrum is obtained by FFT at each probe wavelength and plotted in color scale in Fig. 3(a). It exhibits one intense feature centered at $3.41 \pm 0.02 \mathrm{THz}$, i.e., at the frequency of the $A_{g}$ phonon mode whose calculated real-space representation is depicted in the inset. A closer look at the FFT-spectrum intensity reveals two peculiarities: an enhancement in the exciton-wavelength region and quenchings at $580 \mathrm{~nm}(2.14 \mathrm{eV})$ and $620 \mathrm{~nm}(2.00 \mathrm{eV})$. We argue that the enhancement and quenching of the $A_{g}$ phonon mode intensity point at an interaction between this vibrational mode and the excitonic transition.

The fit parameters of the initial phonon amplitude and phase are plotted on the left axes of Figs. 3(b) and 3(c), respectively. The corresponding $a b$ initio quantities are explicitly calculated and reported on the right axis for direct comparison. As a first result, the phonon phase indicates, at all wavelengths, a cosine-like oscillation of the atoms
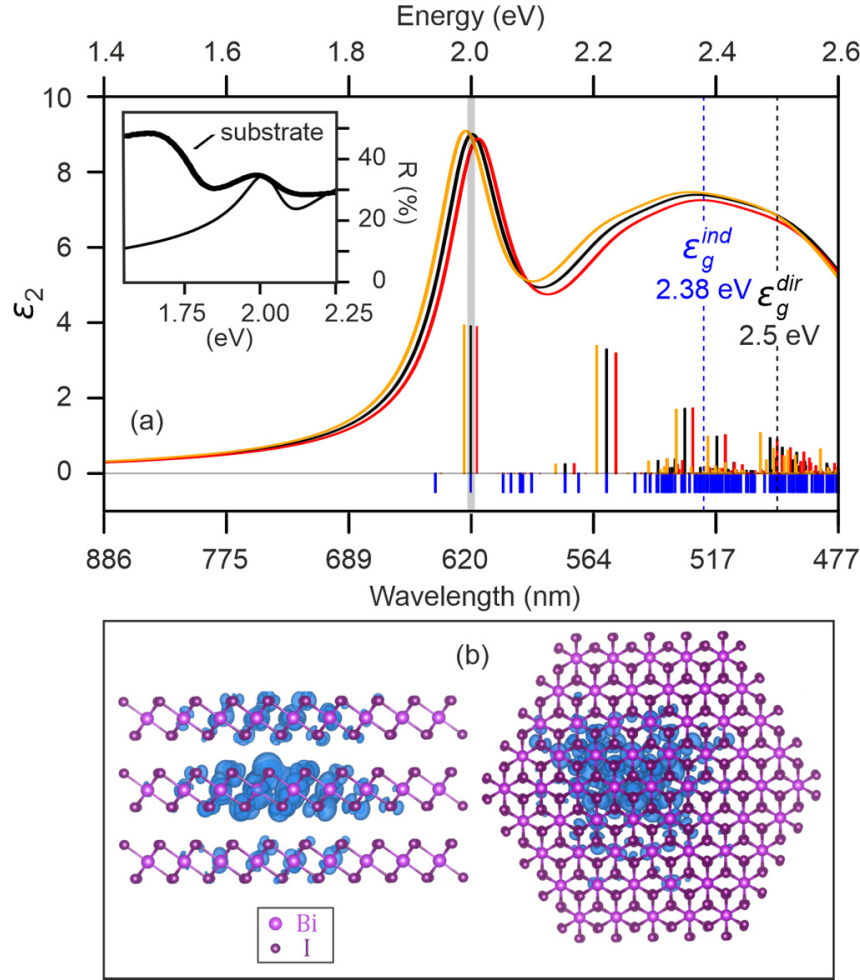

FIG. 2. (a) BSE absorption spectrum of $\mathrm{BiI}_{3}$ computed at equilibrium (black line) and with the atoms displaced along the $A_{g}$ phonon mode with $\pm \Delta_{0}=0.05 \mathrm{Bohr} \approx 2.6 \mathrm{pm}$ (red and orange line). The corresponding equilibrium reflectivity is shown in the inset (thin line) and compared with the experimental measurement (thick line). At low energy, the two differ by the back reflection of the sample holder. The blue vertical lines represent the excitonic energies at equilibrium, while the black, red, and orange bars are the same poles weighted by the oscillator strengths. The blue and black dashed lines

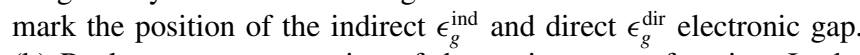
(b) Real-space representation of the exciton wave function. In the side view, three layers of the bulk are shown, with the hole fixed in the central layer.

around their equilibrium position. We deduce that coherent optical phonons are generated via a displacive excitation mechanism, consistent with the full symmetry of the $A_{g}$ vibrational mode and the absorptive excitation regime of our experiment [40-43].

The calculated amplitude and phase are obtained as follows. First, the GW+BSE reflectivity is recomputed with the atoms displaced by an amount $\Delta X_{A_{g}}=\Delta_{0} u_{A_{g}}$ along the direction of the eigenvector $u_{A_{g}}$ of the $A_{g}$ phonon mode [green arrows, inset of Fig. 3(a)]. Assuming that the atoms oscillate according to $X(t)=X_{0}+\Delta X_{A_{g}} \cos \left(\omega_{A_{g}} t\right)$, we obtain the reflectivity, $R^{X}(\omega)$, and thus the reflectivity variation, $F(\omega)=\left(R^{X}-R^{X_{0}}\right) / R^{X_{0}}$, for distinct positions of the displaced atoms. We find that the atomic displacement induces a change in the band gap, $\Delta E_{\text {gap }}$. This affects the dielectric function, and thus, the exciton binding energy which responds to the modified Coulomb screening with a change $\Delta E_{b e}$ in the opposite direction. The resulting shift of the excitonic peak is $\Delta E=\Delta E_{\text {gap }}+\Delta E_{b e}$. The overall picture is of an excitonic 


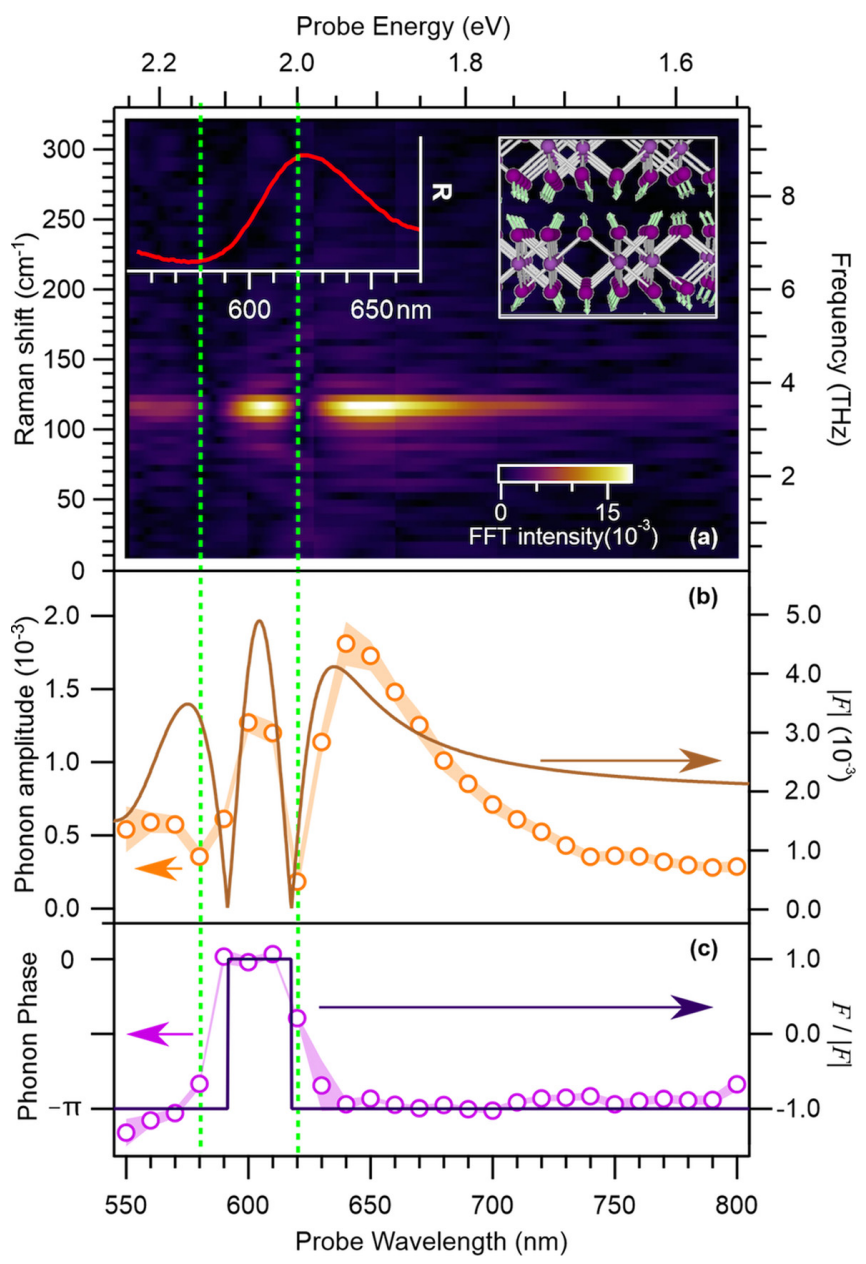

FIG. 3. (a) FFT of the coherent optical response at all probed wavelengths. Left inset: the measured equilibrium reflectance (same as in inset of Fig. 2(a) and in Fig. S8 [34]). Right inset: the realspace representation of the $A_{g}$ phonon mode. (b) Experimental fit parameters of the initial amplitude and (c) phase of the coherent optical phonon as function of the probe wavelength (left axis) and corresponding calculated quantities (right axis).

resonance that oscillates back and forth in energy as shown in Fig. 2(a) (yellow and red spectra).

\section{TRACKING THE PHONON-MEDIATED MODULATION OF THE EXCITONIC RESONANCE}

We now compare the calculated $|F(\omega)|$ and $F(\omega) /|F(\omega)|$ with the measured initial amplitude and phase of the coherent optical response, respectively. Clearly, both quantities confirm the amplitude enhancement as well as the two nodes at the wavelengths of the $\pi$-phase shift (for further details, see also Fig. S7 in Ref. [34]). The node at the exciton wavelength $(620 \mathrm{~nm})$ is due to the opposite sign of the reflectivity variation generated by the shift of the excitonic peak. Concerning the other node, a bunch of excitons with strong exciton-phonon coupling is found to produce a broad peak centered at $525 \mathrm{~nm}(2.35 \mathrm{eV})$. Thus, the second node at $580 \mathrm{~nm}$ results from the combination of the displacements of the two excitonic peaks. The concomitant observation
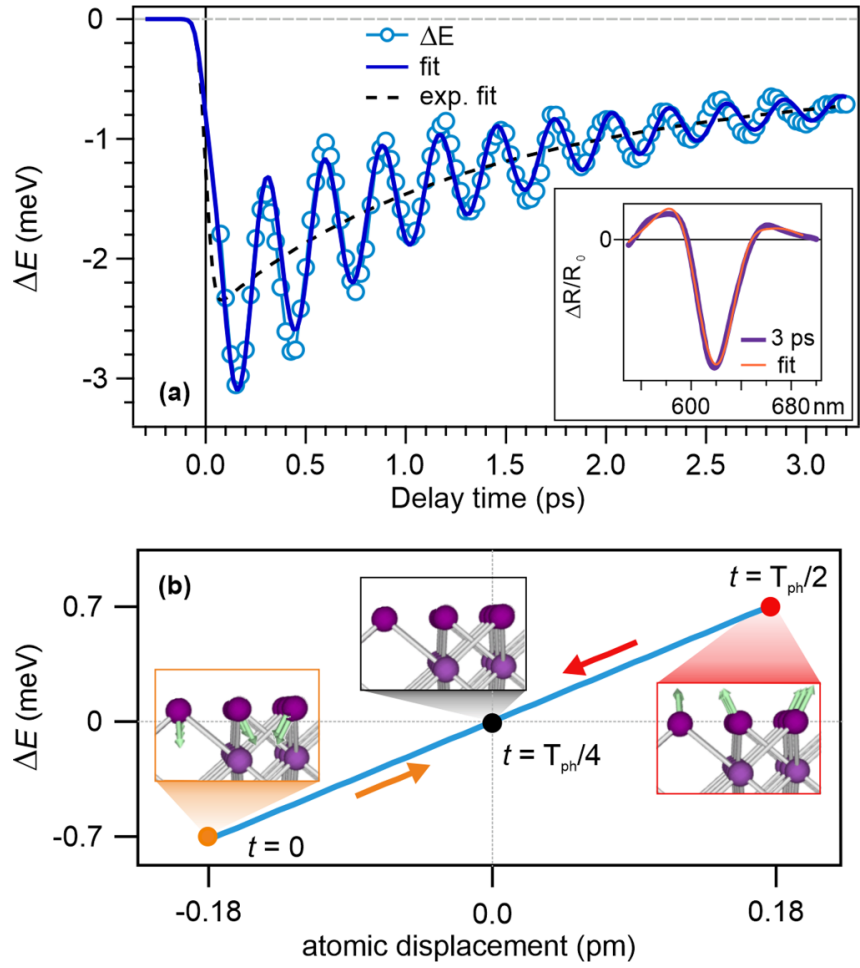

FIG. 4. (a) Energy shift of the excitonic peak (blue circles). The $\Delta E$ fit curve (solid line) is given by a single-exponential decay function (dashed line) multiplied by an exponentially damped cosine function. In inset, spectrum at 3 ps (purple line) and relative differential fit (orange line). (b) The microscopic effect of exciton-phonon coupling: periodic renormalization of the excitonic peak position by the atomic displacement. $T_{\mathrm{ph}}$ refers to the phonon period.

of the predicted amplitude enhancement and $\pi$-phase shift [11] provides unambiguous proof of coupling between coherently activated $A_{g}$ phonons and excitons. Furthermore, by exploiting the excellent agreement between theory and experiment, we provide a quantitative estimate of the photoinduced atomic displacement and the associated energy shifts. An intensity variation of the coherent optical response on the order of $F(\omega) \approx 10^{-3}$ is obtained in the simulations upon atomic displacement in the range of $\Delta_{0} \approx 10^{-1} \mathrm{pm}$. We find that for $\Delta_{0}=0.05 \mathrm{pm}$ the excitonic peak is predicted to shift by $\Delta E=0.2 \mathrm{meV}$ as a result of a upshift of the band gap edge by $\Delta E_{\text {gap }}=0.3 \mathrm{meV}$ and an opposite shift of the binding energy of $\Delta E_{b e}=0.1 \mathrm{meV}$.

To unravel the impact of exciton-phonon coupling on the dynamics of the excitonic resonance, we follow the transient energy position of the excitonic peak. This is obtained by differential fitting of the transient reflectivity spectra [inset of Fig. 4(a)] after parametrization of the equilibrium reflectance (see Ref. [34] for details about the fitting procedure). Figure 4(a) reports the energy shift, $\Delta E$, from the equilibrium position of the Gaussian peak accounting for the excitonic resonance (blue circles). The evaluated temporal window spans up to a few picoseconds to cover the phonon dephasing time. Upon photoexcitation, the excitonic resonance undergoes an instantaneous red shift (within the pump-probe cross correlation) followed by a blue shift toward the equilibrium 
position. The latter evolves on a fast timescale of $1.110 \pm$ 0.075 ps (dashed line), and a slower one that exceeds the evaluated temporal window and is accounted in the fit by an additional offset. The blue shift is superimposed by a periodic energy modulation at the exact frequency of the $A_{g}$-phonon mode, damped on a timescale of $1.420 \pm 0.075$ ps. Both the red and blue shifts are rather common in photoexcited semiconductors and typically relate to band-gap renormalization [44-47] and exciton-binding-energy changes [48,49] induced by dynamical screening, and conduction-band filling by photoexcited carriers [50-52]. Conversely, the coherent modulation of the excitonic resonance has been rarely observed in the photoinduced dynamics of semiconductors with strong exciton-phonon coupling $[11,15,53]$. We explain this fact by noting that a suitable intensity ratio of the coherent and incoherent optical response is required in order to avoid the exciton energy modulation to be buried underneath the photobleaching spectral intensity, as reported, for instance, in Ref. [11]. Our measurements indicate that such ratio is on the order of 1:10 and is achieved by off-resonance photoexcitation with energy above the optical band gap in order to retain from too intense photobleaching of the excitonic resonance.

The periodic modulation of the excitonic peak exhibits an initial amplitude of $\approx 0.7 \mathrm{meV}$ [54] reproduced by the simulation with an atomic displacement of $0.18 \mathrm{pm}$ [see Fig. 4(b)]. The corresponding reflectivity variation is on the expected order of $10^{-3}$, differing only by a factor of $\approx 3$ from the measured phonon amplitude at the respective probe wavelengths [see Fig. 3(b)]. All these findings demonstrate that tracking the energy modulation of the excitonic resonance induced by coherent phonons enables to trace atomic displacements along the direction of the phonon mode. Effectively, our combined experimental and theoretical approach successfully addresses the coherent phonon dynamics coupled to the exciton in real space with subpicometer resolution.

\section{CONCLUSION}

The amplitude enhancement and phase shift of the coherent phonon are established as univocal spectral fingerprints of exciton-phonon coupling. We prove that the excitonic resonance of the layered semiconductor $\mathrm{BiI}_{3}$ can be optically modulated via coupling of coherent atomic vibrations of which we are able to estimate the spatial extent with subpicometer resolution. Note that the present results can be extended to any photoexcited semiconductor with the exciton strongly coupled to a lattice deformation. As a step forward, we propose that ultrafast modulation of excitonic resonances could be experimentally controlled by varying the laser fluence or tuning the pump photon energy across the exciton transition, paving the way toward optical control of the nonequilibrium optoelectronic properties of low-dimensional semiconductors.

\section{ACKNOWLEDGMENTS}

S.P., L.S., and C.G. acknowledge partial support from D.1 and D.2.2 grants of the Universitá Cattolica del Sacro Cuore. C.G. and P.F. acknowledge financial support from MIUR through the project CENTRAL (MIUR (IT), 20172H2SC4_005) within the PRIN 2017 program. D.S. acknowledges the funding received from MIUR PRIN BIOX (MIUR (IT), 20173B72NB), and from the European Union projects: MaX Materials design at the eXascale H2020-INFRAEDI-2018-2020, (European Union, 824143); and Nanoscience Foundries and Fine AnalysisEurope H2020-INFRAIA-2014-2015, (Nanoscience Foundries and Fine AnalysisEurope, 654360). A. M.-S. acknowledges the Ramon y Cajal programme (Grant RYC2018-024024-I; MINECO, Spain).
[1] P. B. Allen, Theory of Thermal Relaxation of Electrons in Metals, Phys. Rev. Lett. 59, 1460 (1987).

[2] G. Sangiovanni, M. Capone, and C. Castellani, Relevance of phonon dynamics in strongly correlated systems coupled to phonons: Dynamical mean-field theory analysis, Phys. Rev. B 73, 165123 (2006).

[3] M. Bauer, A. Marienfeld, and M. Aeschlimann, Hot electron lifetimes in metals probed by time-resolved two-photon photoemission, Prog. Surf. Sci. 90, 319 (2015).

[4] J. Sjakste, K. Tanimura, G. Barbarino, L. Perfetti, and N. Vast, Hot electron relaxation dynamics in semiconductors: Assessing the strength of the electron-phonon coupling from the theoretical and experimental viewpoints, J. Phys.: Condens. Matter 30, 353001 (2018).

[5] S. Hellmann, T. Rohwer, M. Kalläne, K. Hanff, C. Sohrt, A. Stange, A. Carr, M. Murnane, H. Kapteyn, L. Kipp, M. Bauer, and K. Rossnagel, Time-domain classification of charge-density-wave insulators, Nat. Commun. 3, 1069 (2012).

[6] M. Porer, U. Leierseder, J.-M. Ménard, H. Dachraoui, L. Mouchliadis, I. E. Perakis, U. Heinzmann, J. Demsar, K.
Rossnagel, and R. Huber, Non-thermal separation of electronic and structural orders in a persisting charge density wave, Nat. Mater. 13, 857 (2014).

[7] J. Maklar, Y. W. Windsor, C. W. Nicholson, M. Puppin, P. Walmsley, V. Esposito, M. Porer, J. Rittmann, D. Leuenberger, M. Kubli, M. Savoini, E. Abreu, S. L. Johnson, P. Beaud, G. Ingold, U. Staub, I. R. Fisher, R. Ernstorfer, M. Wolf, and L. Rettig, Nonequilibrium charge-density-wave order beyond the thermal limit, Nat. Commun. 12, 2499 (2021).

[8] F. Hüser, T. Olsen, and K. S. Thygesen, How dielectric screening in two-dimensional crystals affects the convergence of excited-state calculations: Monolayer $\mathrm{MoS}_{2}$, Phys. Rev. B 88, 245309 (2013).

[9] B. R. Carvalho, L. M. Malard, J. M. Alves, C. Fantini, and M. A. Pimenta, Symmetry-Dependent Exciton-Phonon Coupling in 2D and Bulk $\mathrm{MoS}_{2}$ observed by resonance Raman scattering, Phys. Rev. Lett. 114, 136403 (2015).

[10] F. Paleari, H. P. C. Miranda, A. Molina-Sánchez, and L. Wirtz, Exciton-Phonon Coupling in the Ultraviolet Absorption and Emission Spectra of Bulk Hexagonal Boron Nitride, Phys. Rev. Lett. 122, 187401 (2019). 
[11] C. Trovatello, H. P. C. Miranda, A. Molina-Sánchez, R. Borrego-Varillas, C. Manzoni, L. Moretti, L. Ganzer, M. Maiuri, J. Wang, D. Dumcenco, A. Kis, L. Wirtz, A. Marini, G. Soavi, A. C. Ferrari, G. Cerullo, D. Sangalli, and S. D. Conte, Strongly coupled coherent phonons in single-layer $\mathrm{MoS}_{2}$, ACS Nano 14, 5700 (2020).

[12] D. Li, C. Trovatello, S. Dal Conte, M. Nuß, G. Soavi, G. Wang, A. C. Ferrari, G. Cerullo, and T. Brixner, Exciton-phonon coupling strength in single-layer $\mathrm{MoSe}_{2}$ at room temperature, Nat. Commun. 12, 954 (2021).

[13] S. Brem, A. Ekman, D. Christiansen, F. Katsch, M. Selig, C. Robert, X. Marie, B. Urbaszek, A. Knorr, and E. Malic, Phonon-assisted photoluminescence from indirect excitons in monolayers of transition-metal dichalcogenides, Nano Lett. 20, 2849 (2020).

[14] H.-Y. Chen, D. Sangalli, and M. Bernardi, Exciton-Phonon Interaction and Relaxation Times from First Principles, Phys. Rev. Lett. 125, 107401 (2020).

[15] J. Fu, M. Li, A. Solanki, Q. Xu, Y. Lekina, S. Ramesh, Z. X. Shen, and T. C. Sum, Electronic states modulation by coherent optical phonons in 2D halide perovskites, Adv. Mater. 33, 2006233 (2021).

[16] A. Molina-Sánchez, D. Sangalli, L. Wirtz, and A. Marini, $A b$ initio calculations of ultrashort carrier dynamics in twodimensional materials: Valley depolarization in single-layer $\mathrm{WSe}_{2}$, Nano Lett. 17, 4549 (2017).

[17] H. P. C. Miranda, S. Reichardt, G. Froehlicher, A. MolinaSánchez, S. Berciaud, and L. Wirtz, Quantum interference effects in resonant Raman spectroscopy of single- and triplelayer $\mathrm{MoTe}_{2}$ from first-principles, Nano Lett. 17, 2381 (2017).

[18] A. K. Ganguly and J. L. Birman, Theory of lattice Raman scattering in insulators, Phys. Rev. 162, 806 (1967).

[19] A. S. Rury, S. Soreson, E. Driscoll, and J. M. Dawlaty, Electronic state-resolved electronphonon coupling in an organic charge transfer material from broadband quantum beat spectroscopy, J. Phys. Chem. Lett. 6, 3560 (2015).

[20] L. Ni, U. Huynh, A. Cheminal, T. H. Thomas, R. Shivanna, T. F. Hinrichsen, S. Ahmad, A. Sadhanala, and A. Rao, Real-time observation of exciton-phonon coupling dynamics in self-assembled hybrid perovskite quantum wells, ACS Nano 11, 10834 (2017).

[21] F. Giustino, Electron-phonon interactions from first principles, Rev. Mod. Phys. 89, 015003 (2017).

[22] G. Onida, L. Reining, R. W. Godby, R. Del Sole, and W. Andreoni, Ab Initio Calculations of the Quasiparticle and Absorption Spectra of Clusters: The Sodium Tetramer, Phys. Rev. Lett. 75, 818 (1995).

[23] P. Cudazzo, First-principles description of the exciton-phonon interaction: A cumulant approach, Phys. Rev. B 102, 045136 (2020).

[24] H. Katsuki, J. C. Delagnes, K. Hosaka, K. Ishioka, H. Chiba, E. S. Zijlstra, M. E. Garcia, H. Takahashi, K. Watanabe, M. Kitajima, Y. Matsumoto, K. G. Nakamura, and K. Ohmori, Alloptical control and visualization of ultrafast two-dimensional atomic motions in a single crystal of bismuth, Nat. Commun. 4 , 2801 (2013).

[25] M. Marsili, A. Molina-Sánchez, M. Palummo, D. Sangalli, and A. Marini, Spinorial formulation of the $G W$-BSE equations and spin properties of excitons in two-dimensional transition metal dichalcogenides, Phys. Rev. B 103, 155152 (2021).
[26] A. J. Lehner, H. Wang, D. H. Fabini, C. Liman, C. Hébert, E. Perry, G. Wang, M. Bazan, M. Chabinyc, and R. Seshadri, Electronic structure and photovoltaic application of $\mathrm{BiI}_{3}$, Appl. Phys. Lett. 107, 131109 (2015).

[27] Z. Shi, J. Guo, Y. Chen, Q. Li, Y. Pan, H. Zhang, Y. Xia, and W. Huang, Lead-free organicinorganic hybrid perovskites for photovoltaic applications: Recent advances and perspectives, Adv. Mater. 29, 1605005 (2017).

[28] Y. Kaifu, Excitons in layered $\mathrm{BiI}_{3}$ single crystals, J. Lumin. 42, 61 (1988).

[29] A. Nila, A. Matea, M. Baibarac, and I. Baltog, The exciton-phonon interaction as stimulated Raman scattering effect supported by the excitonic photoluminescence in $\mathrm{BiI}_{3}$ layered crystal structure, J. Lumin. 182, 166 (2017).

[30] A. Saitoh, T. Komatsu, and T. Karasawa, Exciton-phonon interaction and conversion of excitons from free to self-trapped states in layered metal iodide crystals under hydrostatic pressure, J. Lumin. 87-89, 633 (2000).

[31] T. Karasawa, T. Komatsu, K. Miyata, T. Iida, and Y. Kaifu, Stacking disorder effects on resonance Raman scattering in $\mathrm{BiI}_{3}$, Physica B+C (Amsterdam) 105, 88 (1981).

[32] D. Tiwari, D. Alibhai, and D. J. Fermin, Above $600 \mathrm{mV}$ open-circuit voltage $\mathrm{Bii}_{3}$ solar cells, ACS Energy Lett. 3, 1882 (2018).

[33] M. Scholz, K. Oum, and T. Lenzer, Pronounced exciton and coherent phonon dynamics in $\mathrm{BiI}_{3}$, Phys. Chem. Chem. Phys. 20, 10677 (2018).

[34] See Supplemental Material at http://link.aps.org/supplemental/ 10.1103/PhysRevResearch.3.043175 for details about the experimental details, the theoretical model, the sample characterization and the data analysis.

[35] R. E. Brandt, R. C. Kurchin, R. L. Z. Hoye, J. R. Poindexter, M. W. B. Wilson, S. Sulekar, F. Lenahan, P. X. T. Yen, V. Stevanovi, J. C. Nino, M. G. Bawendi, and T. Buonassisi, Investigation of bismuth triiodide $\left(\mathrm{BiI}_{3}\right)$ for photovoltaic applications, J. Phys. Chem. Lett. 6, 4297 (2015).

[36] The exciton binding energy reported in the literature is 160 $180 \mathrm{meV}$ [28]. This estimate is based on the hydrogen model accounting for the macroscopic screening. The present calculations account for both macroscopic and microscopic screening effects.

[37] T. Habe and K. Nakamura, Optical properties of monolayer, multilayer, and bulk $\mathrm{BiI}_{3}$ studied using time-dependent density functional theory, Phys. Rev. B 103, 115409 (2021).

[38] G. E. Jellison, J. O. Ramey, and L. A. Boatner, Optical functions of $\mathrm{BiI}_{3}$ as measured by generalized ellipsometry, Phys. Rev. B 59, 9718 (1999).

[39] N. J. Podraza, W. Qiu, B. B. Hinojosa, H. Xu, M. A. Motyka, S. R. Phillpot, J. E. Baciak, S. Trolier-McKinstry, and J. C. Nino, Band gap and structure of single crystal $\mathrm{BiI}_{3}$ : Resolving discrepancies in literature, J. Appl. Phys. 114, 033110 (2013).

[40] T. K. Cheng, J. Vidal, H. J. Zeiger, G. Dresselhaus, M. S. Dresselhaus, and E. P. Ippen, Mechanism for displacive excitation of coherent phonons in $\mathrm{Sb}, \mathrm{Bi}, \mathrm{Te}$, and $\mathrm{Ti}_{2} \mathrm{O}_{3}$, Appl. Phys. Lett. 59, 1923 (1991).

[41] H. J. Zeiger, J. Vidal, T. K. Cheng, E. P. Ippen, G. Dresselhaus, and M. S. Dresselhaus, Theory for displacive excitation of coherent phonons, Phys. Rev. B 45, 768 (1992). 
[42] K. Ishioka, M. Kitajima, and O. V. Misochko, Temperature dependence of coherent $\mathrm{A}_{1 g}$ and $\mathrm{E}_{g}$ phonons of bismuth, J. Appl. Phys. 100, 093501 (2006).

[43] K. Ishioka and O. V. Misochko, Progress in Ultrafast Intense Laser Science, edited by K. Yamanouchi, A. Giulietti, and K. Ledingham (Springer, Berlin, 2010) Vol. 5, pp. 23-46.

[44] S. Pagliara, G. Galimberti, S. Mor, M. Montagnese, G. Ferrini, M. S. Grandi, P. Galinetto, and F. Parmigiani, Photoinduced $\pi-\pi^{*}$ band gap renormalization in graphite, J. Am. Chem. Soc. 133, 6318 (2011).

[45] A. Chernikov, C. Ruppert, H. Hill, A. F. Rigosi, and T. F. Heinz, Population inversion and giant bandgap renormalization in atomically thin $\mathrm{WS}_{2}$ layers, Nat. Photon. 9, 466 (2015).

[46] E. A. A. Pogna, M. Marsili, D. De Fazio, S. Dal Conte, C. Manzoni, D. Sangalli, D. Yoon, A. Lombardo, A. C. Ferrari, A. Marini, G. Cerullo, and D. Prezzi, Photo-induced bandgap renormalization governs the ultrafast response of single-layer $\mathrm{MoS}_{2}$, ACS Nano 10, 1182 (2016).

[47] S. Mor, M. Herzog, D. Golež, P. Werner, M. Eckstein, N. Katayama, M. Nohara, H. Takagi, T. Mizokawa, C. Monney, and J. Stähler, Ultrafast Electronic Band Gap Control in an Excitonic Insulator, Phys. Rev. Lett. 119, 086401 (2017).

[48] P. D. Cunningham, A. T. Hanbicki, K. M. McCreary, and B. T. Jonker, Photoinduced bandgap renormalization and exciton binding energy reduction in ws2, ACS Nano 11, 12601 (2017).
[49] T. Jiang, R. Chen, X. Zheng, Z. Xu, and Y. Tang, Photo-induced excitonic structure renormalization and broadband absorption in monolayer tungsten disulphide, Opt. Express 26, 859 (2018).

[50] B. Ullrich, H. Xi, and J. S. Wang, Photoinduced band filling in strongly confined colloidal PbS quantum dots, J. Appl. Phys. 115, 233503 (2014).

[51] P. Franceschini, L. Carletti, A. P. Pushkarev, F. Preda, A. Perri, A. Tognazzi, A. Ronchi, G. Ferrini, S. Pagliara, F. Banfi, D. Polli, G. Cerullo, C. De Angelis, S. V. Makarov, and C. Giannetti, Tuning the ultrafast response of Fano resonances in halide perovskite nanoparticles, ACS Nano 14, 13602 (2020).

[52] G. Jnawali, Y. Xiang, S. M. Linser, I. A. Shojaei, R. Wang, G. Qiu, C. Lian, B. M. Wong, W. Wu, P. D. Ye, Y. Leng, H. E. Jackson, and L. M. Smith, Ultrafast photoinduced band splitting and carrier dynamics in chiral tellurium nanosheets, Nat. Commun. 11, 3991 (2020).

[53] E. Baldini, A. Dominguez, T. Palmieri, O. Cannelli, A. Rubio, P. Ruello, and M. Chergui, Exciton control in a room temperature bulk semiconductor with coherent strain pulses, Sci. Adv. 5, eaax2937 (2019).

[54] We note that a periodic modulation of the transient reflectivity minimum is already visible by eye. The high statistics of our transient reflectivity data allows us to resolve relative energy shifts of only few tens of meV which are robust against the choice of fit analysis. 